\title{
The Logarithmic Sobolev Inequality for Discrete Spin Systems on a Lattice ${ }^{\star}$
}

\author{
Daniel W. Stroock ${ }^{1}$ and Boguslaw Zegarlinski ${ }^{1,2}$ \\ ${ }^{1}$ Department of Mathematics, 2-272, M.I.T. Cambridge, MA 02139, USA \\ ${ }^{2}$ Fakultät für Mathematik, Ruhr-Universität-Bochum, W-4630 Bochum 1, Postfach 102148, \\ Germany
}

Received February 15, 1992

\begin{abstract}
For finite range lattice gases with a finite spin space, it is shown that the Dobrushin-Shlosman mixing condition is equivalent to the existence of a logarithmic Sobolev inequality for the associated (unique) Gibbs state. In addition, implications of these considerations for the ergodic properties of the corresponding Glauber dynamics are examined.
\end{abstract}

\section{Preliminaries}

We begin by introducing the setting in which and some of the notation with which we will be working throughout.

The Lattice. The lattice $\Gamma$ underlying our model will be the $d$-dimensional square lattice $\mathbb{Z}^{d}$ for some fixed $d \in \mathbb{Z}^{+}$, and, for $\mathbf{j} \in \Gamma$, we will use the norm $|\mathbf{k}| \equiv \max _{1 \leqq i \leqq d}$ $\left|\mathbf{k}^{i}\right|$. Given $\Lambda \subseteq \Gamma$, we will use $\Lambda \complement \equiv \Gamma \backslash \Lambda$ to denote the complement of $\Lambda,|\Lambda|$ to denote the cardinality of $\Lambda$, and $\mathbf{j}+\Lambda$ to denote the translate $\{\mathbf{j}+\mathbf{k}: \mathbf{k} \in \Lambda\}$ of $\Lambda$ by $\mathbf{j} \in \Gamma$. Furthermore, for each $R \in \mathbb{R}^{+}$, we take the $R$-boundary $\partial_{R} \Lambda$ to be the set

$$
\{\mathbf{k} \in \Lambda \mathfrak{l}:|\mathbf{k}-\mathbf{j}| \leqq R \text { for some } \mathbf{j} \in \Lambda\} \text {. }
$$

We will often use the notation $\Lambda \Subset \Gamma$ to mean that $|\Lambda|<\infty$, and $\mathfrak{F}$ will stand for the set of all non-empty $\Lambda \Subset \Gamma$. A monotone sequence $\mathfrak{F}_{0} \equiv\left\{\Lambda_{n}: n \in \mathbb{N}\right\} \subseteq \mathfrak{F}$ will be called a countable exhaustion if $\Lambda_{n}>\Gamma$.

The Spin Space. The single spin space for our model will be a finite set $Q$ with the topology of all subsets, corresponding Borel field $\mathscr{B}_{Q}$, and normalized uniform measure $v_{0}$ on $\left(Q, \mathscr{B}_{Q}\right)$. Given a real-valued function $f$ on $Q$, we define the differential $\partial f$ of $f$ by

$$
\partial f \equiv f-v_{0} f,
$$

where we have introduced the notation $\mu \varphi$ (to be used throughout) as one of the various expressions for the integral of a $\mu$-integrable function $\varphi$ with respect to a measure $\mu$.

* During the period of this research, both authors were partially supported by NSF grant DMS 8913328 
The Configuration Space. Given any non-empty subset $\Lambda$ of $\Gamma$, we give $Q^{\Lambda}$ the product topology and use $\mathscr{B}_{Q^{A}}$ to denote the associated Borel field. In particular, our configuration space will be the space $\Omega \equiv Q^{\Gamma}$, and, for each $\emptyset \neq \Lambda \subseteq \Gamma$, $\omega \in \Omega \mapsto \omega_{\Lambda} \in Q^{\Lambda}$ will denote the natural projection mapping from $\Omega$ onto $Q^{\Lambda}, \mathscr{F}_{\Lambda}$ and $\mathfrak{U}_{\Lambda}(\Omega)$ will denote the $\sigma$-algebra of sets of the form $\left\{\omega \in \Omega: \omega_{\Lambda} \in A\right\}$ with $A \in \mathscr{B}_{Q^{\Lambda}}$ and the space of bounded, $\mathscr{F}_{\Lambda}$-measurable functions $\varphi: \Omega \rightarrow \mathbb{R}$, and $C_{\Lambda}(\Omega)$ will stand for the continuous elements of $\mathfrak{U}_{\Lambda}(\Omega)$. When $\Lambda=\{\mathbf{k}\}$, we will use $\omega_{\mathbf{k}}$, in place of $\omega_{\{\mathbf{k}\}}$; and when $\Lambda=\Gamma$, we will drop the subscript entirely. Thus, for example, $\mathfrak{A}(\Omega)$ and $C(\Omega)$ are, respectively, the space of bounded, $\mathscr{B}_{\Omega}$-measurable functions and the subspace of continuous elements of $\mathfrak{U}(\Omega)$. Also, we will say that $\varphi \in \mathfrak{A}(\Omega)$ is local and will write $\varphi \in \mathfrak{U}_{0}(\Omega)$ if $\varphi \in \mathfrak{A}_{\Lambda}$ for some $\Lambda \in \mathfrak{F}$ (notice that, because $Q$ is finite, all elements of $\mathfrak{A}_{0}(\Omega)$ are necessarily continuous); and, for any $\varphi: \Omega \rightarrow \mathbb{R},\|\varphi\|_{\text {u }}$ will be used to denote its uniform (i.e. the "sup") norm, and the standard notion of convergence of functions in $C(\Omega)$ will be the one induced by $\|\cdot\|_{\mathbf{u}}$. On the other hand, the standard notion of convergence for measures will be that of weak convergence. Thus, for example, if $\emptyset \neq \Lambda \subseteq \Gamma$ and $\left\{\mu_{n}\right\}_{1}^{\infty}$ is a sequence from $\mathfrak{M}_{1}\left(Q^{\Lambda}\right)$ (i.e., the set of Borel probability measures on $Q^{\Lambda}$ ) then we say that $\mu_{n}$ converges to $\mu$ and will write $\mu_{n} \Rightarrow \mu$ if $\mu_{n} \varphi \rightarrow \mu \varphi$ for every continuous $\varphi: Q^{\Lambda} \rightarrow \mathbb{R}$. Also, given $\mu \in \mathfrak{M}_{1}(\Omega)$ and $\emptyset \neq \Lambda \subseteq \Gamma$, we use $\mu_{\Lambda} \in \mathfrak{M}_{1}\left(Q^{\Lambda}\right)$ to denote the marginal distribution of $\omega \in \Omega \mapsto \omega_{\Lambda} \in Q^{\Lambda}$ under $\mu$. That is, if $\varphi \in \mathfrak{U}_{\Lambda}$ and $\varphi_{\Lambda}$ is the Borel measurable function on $Q^{\Lambda}$ determined by $\varphi(\omega)=\varphi_{\Lambda}\left(\omega_{\Lambda}\right)$, then $\mu_{A}$ is the element of $\mathfrak{M}_{1}\left(Q^{\Lambda}\right)$ for which $\mu_{\Lambda} \varphi_{\Lambda}=\mu \varphi$. In keeping with our use of $\mu f$ to denote the integral of a function $f$ with respect to a measure $\mu$, we will use

$$
\mu(f, g) \equiv \mu(f g)-\mu(f) \mu(g)
$$

to denote the covariance of two functions $f$ and $g$ from $L^{2}(\mu)$.

Finally, for each $\mathbf{k} \in \Gamma$, we define the shift transformation $\theta^{\mathbf{k}}: \Omega \rightarrow \Omega$ so that $\left(\theta^{\mathbf{k}} \omega\right)_{\mathbf{j}}=\omega_{\mathbf{k}+\mathbf{j}}$ for every $\omega \in \Omega$ and every $\mathbf{j} \in \Gamma$.

The Standard Gradient Operation. In order to describe a discrete gradient operator on $\Omega$, it will be convenient to introduce additional notation. In the first place, given $\emptyset \neq \Lambda \subseteq \Gamma$, we define

$$
\left(x^{\Lambda}, y^{\Lambda \complement}\right) \in Q^{\Lambda} \times Q^{\Lambda \complement} \mapsto x^{\Lambda} \cdot y^{\Lambda \complement \in \Omega}
$$

so that $x^{\Lambda} \cdot y^{\Lambda \complement}$ is the element $\omega \in \Omega$ determined by

$$
\omega_{\Lambda}=x^{\Lambda} \text { and } \omega_{\Lambda \uparrow}=y^{\Lambda \uparrow} ;
$$

and, for $f: \Omega \rightarrow \mathbb{R}$ and $y^{\Lambda \complement} \in Q^{\Lambda \uparrow}$, we define $f\left(\cdot \mid y^{\Lambda}\right)$ on $Q^{\Lambda}$ and $f_{\Lambda}\left(\cdot \mid y^{\Lambda \uparrow}\right)$ on $\Omega$ by

$$
x^{\Lambda} \in Q^{\Lambda} \mapsto f\left(x^{\Lambda} \mid y^{\Lambda}\right) \equiv f\left(x^{\Lambda} \cdot y^{\Lambda \complement}\right)
$$

and

$$
\omega \in \Omega \mapsto f_{\Lambda}\left(\omega \mid y^{\Lambda \complement}\right) \equiv f\left(\omega_{\Lambda} \cdot y^{\Lambda \complement}\right) .
$$

Secondly, for $\omega \in \Omega$, we write $f_{\Lambda}(\cdot \mid \omega)$ instead of $f_{\Lambda}\left(\cdot \mid \omega_{\Lambda \uparrow}\right)$; and, when $\Lambda=\{\mathbf{k}\}$ we will use $f_{\mathbf{k}}(\cdot \mid \omega)$ in place of $f_{\{\mathbf{k}\}}(\cdot \mid \omega)$. Since both

$$
\left(x^{\Lambda}, y^{\Lambda \complement}\right) \in Q^{\Lambda} \times Q^{\Lambda \complement} \mapsto x^{\Lambda} \cdot y^{\Lambda \complement} \in \Omega \quad \text { and } \quad(\eta, \omega) \in \Omega^{2} \mapsto \eta_{\Lambda} \cdot \omega_{\Lambda \uparrow} \in \Omega
$$

are continuous maps, all the preceding constructions preserve both continuity and measurability. 
For $f \in \mathfrak{A}$ and non-empty $\Lambda \subseteq \Gamma$, we define the gradient $\left(\nabla_{\Lambda} f\right)$ with respect to the coordinates in the set $\Lambda$ by setting

$$
\left(\nabla_{\Lambda} f(\omega)\right)_{\mathbf{k}}=\left(\partial f_{\mathbf{k}}(\cdot \mid \omega)\right)\left(\omega_{\mathbf{k}}\right) \text { for } \mathbf{k} \in \Lambda
$$

In keeping with our earlier conventions, we take $\nabla=\nabla_{\Gamma}$ and $\nabla_{\mathbf{k}}=\nabla_{\{\mathbf{k}\}}$. Also,

$$
\left|\nabla_{A} f\right|^{2}(\omega) \equiv \sum_{\mathbf{k} \in A}\left|\nabla f_{\mathbf{k}}(\cdot \mid \omega)\right|^{2}\left(\omega_{\mathbf{k}}\right) \in[0, \infty]
$$

Finally, we also introduce the semi-norm

$$
\|f\| \mid \equiv \sum_{\mathbf{k} \in \Gamma}\left\|\nabla_{k} f(\omega)\right\|_{\mathbf{u}} \in[0, \infty]
$$

and define $C^{1}(\Omega)$ to be the space of continuous functions $f$ for which $\|f\| \mid<\infty$.

The Standard Logarithmic Sobolev Inequality. For any non-empty $\Lambda \subseteq \Gamma$ and $\mu \in \mathfrak{M}_{1}(\Omega)$, we define the (standard) logarithmic Sobolev constant $c(\mu ; \Lambda)$ of $\mu$ on $\Lambda$ to be the smallest $c \in[0, \infty]$ with the property that

$$
\text { SLS } \quad \mu f^{2} \log |f| \leqq c \mu\left|\nabla_{\Lambda} f\right|^{2}+\|f\|_{L^{2}(\mu)}^{2} \log \|f\|_{L^{2}(\mu)}, \quad f \in \mathfrak{A}_{\Lambda} .
$$

When $c(\mu ; \Lambda)<\infty$, we say that $\mu$ admits a standard logarithmic Sobolev inequality on $\Lambda$, in which case, SLS with $c=c(\mu ; \Lambda)$ is the standard logarithmic Sobolev inequality for $\mu$ on $\Lambda$; and when $\Lambda=\Gamma$, we drop all reference to $\Lambda$ in the notation. Thus, $c(\mu)=c(\mu ; \Gamma)$.

Local Specifications and Gibbs States. A local specification is a family $\mathbb{E} \equiv\left\{E_{\dot{\Lambda}}\right\}_{\Lambda \in \mathbb{F}}$ which consists of transition probability functions

$$
\Omega \ni \omega \mapsto E_{\Lambda}^{\omega} \in \mathfrak{M}_{1}(\Omega), \quad \Lambda \in \mathfrak{F},
$$

satisfying $E_{\Lambda} f \in \mathfrak{U}_{\Lambda \uparrow}(\Omega)$ for all $f \in \mathfrak{U}$ and the consistency condition

$$
E_{\Lambda^{\prime}}=E_{\Lambda^{\prime}} \circ E_{\Lambda} \text { whenever } \Lambda \subseteq \Lambda^{\prime},
$$

where we have introduced the notation $E_{\Lambda}$ to denote the operator $E_{A}: \mathfrak{A}(\Omega) \rightarrow$ $\mathfrak{U}_{\Lambda \complement}(\Omega)$ given by $E_{\Lambda} \varphi(\omega)=E_{\Lambda}^{\omega} \varphi$. When $\mathfrak{E}$ admits an $R \in \mathbb{R}$ with the property that

$$
\Omega \ni \omega \mapsto E_{\Lambda}^{\omega}(f) \text { is } \mathscr{F}_{\hat{o}_{R} \Lambda} \text {-measurable for every } f \in \mathfrak{A}_{\Lambda} \text { and } \Lambda \in \mathfrak{F},
$$

we say that $\mathbb{E}$ is a local specification with range $R$; and when

$$
E_{\mathbf{k}+\Lambda}\left(f \circ \theta^{\mathbf{k}}\right)=\left(E_{\Lambda} f\right) \circ \theta^{\mathbf{k}} \quad \text { for all } f \in \mathfrak{A}(\Omega), \mathbf{k} \in \Gamma \text {, and } \Lambda \in \mathfrak{F},
$$

we say that $\mathfrak{E}$ is a shift-invariant local specification. Given a local specification $\mathfrak{E}$, we will say that $\mu \in \mathfrak{M}_{1}(\Omega)$ is a Gibbs state for $\mathfrak{E}$ and will write $\mu \in \mathfrak{G}(\mathfrak{E})$ if, for every $\Lambda \in \mathfrak{F}, \omega \in \Omega \mapsto E_{\Lambda}^{\omega} \in \mathfrak{M}_{1}(\Omega)$ is a regular conditional probability distribution of $\mu$ given $\mathfrak{F}_{\Lambda \mathfrak{l}}$. That is, $\mu \in(\mathfrak{G}(\mathfrak{E})$ if and only if it satisfies the Dobrushin-LanfordRuelle condition

DLR

$$
\mu\left(E_{\Lambda} f\right)=\mu(f) \text { for all } \Lambda \in \mathfrak{F} \text { and } f \in \mathfrak{A}(\Omega) ;
$$

Clearly $\left(\mathfrak{b}(\mathfrak{E})\right.$ is convex. Moreover, if $C(\Omega)$ is invariant under $E_{\Lambda}$ for each $\Lambda \in \mathfrak{F}$, then it is an easy matter to show that $\mathfrak{G}(\mathfrak{E})$ is non-empty and compact. In particular, this will be the case when $\mathfrak{E}$ has finite range.

In this paper, our local specification will come from a shift invariant, finite range Gibbs potential $\Phi \equiv\left\{\Phi_{X}\right\}_{X \in \mathfrak{F}}$. That is, 
(1) for each $X \in \mathfrak{F}, \Phi_{X} \in C_{X}(\Omega)$;

(2) for each $\mathbf{k} \in \Gamma$ and $X \in \mathfrak{F}, \Phi_{\mathbf{k}+X}=\Phi_{X}{ }^{\circ} \theta^{\mathbf{k}}$;

(3) there is an $R \in \mathbb{N}$ (the range of $\Phi$ ) such that $\Phi_{X} \equiv 0$ whenever $X \in \mathfrak{F}$ and the diameter of $X$ is greater than $R$;

and $E_{\Lambda}^{\omega}$ is determined from $\Phi$ by

$$
E_{\Lambda}^{\omega} \varphi=\frac{1}{Z_{\Lambda}(\omega)} \int_{Q^{\Lambda}} \varphi\left(x^{\Lambda} \mid \omega\right) e^{-U_{\Lambda}\left(x^{\Lambda} \mid \omega\right)} v_{0}^{\Lambda}\left(d x^{\Lambda}\right),
$$

where

$$
U_{\Lambda}\left(x^{\Lambda} \mid \omega\right) \equiv \sum_{\substack{X \in \mathscr{F} \\ X \cap \Lambda \neq \emptyset}} \Phi_{X}\left(x^{\Lambda} \cdot \omega_{\Lambda \uparrow}\right) \quad \text { for }\left(x^{\Lambda}, \omega\right) \in Q^{\Lambda} \times \Omega,
$$

and $Z_{\Lambda}(\omega)$ is chosen so that $E_{\Lambda}^{\omega}$ is a probability measure. Obviously, the condition (3) above guarantees that the corresponding local specification $\mathfrak{E}$ has range $R$, and therefore we know that $\mathfrak{G}(\Phi) \equiv \mathfrak{G}(\mathfrak{E})$ is a non-empty, compact, and convex.

Dobrushin-Shlosman Conditions. Let $\Phi$ be a shift-invariant, finite range Gibbs potential, and let $\mathbb{E}$ be the corresponding local specification. By the preceding remarks, we know that $\mathfrak{5}(\Phi)$ is necessarily non-empty, compact, and convex. In order to provide a criterion which guarantees uniqueness, Dobrushin and Shlosman introduced what we will call the Dobrushin-Shlosman uniqueness condition, namely: there exists a $Y \in \mathfrak{F}$ and a matrix $\left\{\alpha_{\mathbf{j}, \mathbf{k}}: \mathbf{j} \in Y\right.$ and $\left.\mathbf{k} \notin Y\right\} \subseteq[0, \infty)$ such that, for each $f \in \mathfrak{U}(\Omega)$ :

$\operatorname{DSU}(\mathrm{Y})$

$$
\left\|\partial_{\mathbf{k}} E_{Y} f-E_{Y} \partial_{\mathbf{k}} f\right\|_{\mathbf{u}} \leqq \sum_{\mathbf{j} \in Y} \alpha_{\mathbf{j}, \mathbf{k}}\left\|\partial_{\mathbf{j}} f\right\|_{\mathbf{u}} \quad \text { with }
$$

$$
\sum_{\mathbf{k} \notin Y} \sum_{\mathbf{j} \in Y} \alpha_{\mathbf{j}, \mathbf{k}}=\gamma|Y| \text { for some } \gamma \in(0,1) \text {. }
$$

In fact, what Dobrushin and Shlosman showed (cf. [Dob \& S, 1]) is that DSU(Y) implies the existence of a constant $M \in(0, \infty)$ with the property that, for each $S \in \mathfrak{F}$, one can find a constant $C_{S} \in[0, \infty)$ for which

\section{DSU}

$$
\operatorname{osc}_{\mathrm{k}}\left(E_{\Lambda} f\right) \equiv \sup _{(\eta, \omega) \in \Omega^{2}(\mathbf{k})}\left|E_{\Lambda}^{\eta}(f)-E_{\Lambda}^{\omega}(f)\right| \leqq C_{S}|\|f\|| e^{-M d(S, \Lambda \ell)}
$$

$$
\text { for all } \mathfrak{F} \ni \Lambda \supseteq S, f \in \mathfrak{A}_{S} \text {, and } \mathbf{k} \in \partial_{R} \Lambda \text {, }
$$

where $\Omega^{2}(\mathbf{k}) \equiv\left\{(\eta, \omega) \in \Omega^{2}: \eta_{\mathbf{j}}=\omega_{\mathbf{j}}\right.$ for all $\left.\mathbf{j} \neq \mathbf{k}\right\}$ and $d(S, \Lambda \uparrow)$ denotes the distance from $S$ to the complement of $\Lambda$. Since it is obvious that DSU is more than enough to guarantee that, for each choice of $f \in \mathfrak{U}(\Omega)$ and $\mathfrak{G}(\Phi), \mathfrak{F} \ni \Lambda_{n} \nearrow \Gamma$ and $\left\{\omega_{n}\right\}_{1}^{\infty} \subseteq \Omega$ :

$$
\mu f=\lim _{n \rightarrow \infty} \mu\left(E_{\Lambda_{n}} f\right)=\lim _{n \rightarrow \infty} E_{\Lambda_{n}}^{\omega_{n}} f,
$$

it is clear that DSU, and therefore $\operatorname{DSU}(\mathrm{Y})$, implies that $(5)(\Phi)$ contains precisely one element $\mu_{\Phi}$. Further, the condition DSU(Y) is stable under perturbations in the sense that it holds for all sufficiently small perturbations of $\Phi$ as soon as it holds for $\Phi$ itself. On the other hand, it does not imply that the Gibbs state $\mu_{\Phi}$ depends in an analytic fashion on the perturbation parameter. For this reason, the same authors introduced (cf. [Dob\& S, 2]) a stronger condition, referred to in our articles $[\mathrm{SZ}, 1]$ and $[\mathrm{SZ}, 2]$ as the Dobrushin-Shlosman mixing condition, in which 
$\mathrm{DSU}(\mathrm{Y})$ is replaced by:

$\operatorname{DSM}(\mathrm{Y})$

$$
\left\|\partial_{\mathbf{k}} E_{X} f-E_{X} \partial_{\mathbf{k}} f\right\|_{\mathbf{u}} \leqq \sum_{\mathbf{j} \in X} \alpha_{\mathbf{j}, \mathbf{k}}\left\|\partial_{\mathbf{j}} f\right\|_{\mathbf{u}} \quad \text { for all } \mathfrak{F} \ni X \subseteq Y \text { and } \mathbf{k} \notin X,
$$

$$
\text { where } \sum_{\mathbf{k} \notin Y} \sum_{\mathbf{j} \in Y} \alpha_{j, \mathbf{k}}=\gamma|Y| \text { for some } \gamma \in(0,1) \text {. }
$$

Obviously, DSM(Y) implies DSU(Y) and therefore the uniqueness of the Gibbs state $\mu_{\Phi}$. Moreover, as Dobrushin and Shlosman show in [Dob \& S, 2], DSM(Y) admits a long list equivalent formulations. For our purposes, the most important of these equivalent formulations (cf. Corollary 2.2 below) is the following strengthening of DSU:

DSM

$$
\operatorname{osc}_{\mathbf{k}}\left(E_{\Lambda} f\right) \leqq C_{S}\|f\| e^{-M d(\mathbf{k}, S)} \quad \text { for all } \mathfrak{F} \ni \Lambda \supseteq S, f \in \mathfrak{A}_{S}(\Omega),
$$

and $\mathbf{k} \in \partial_{R} \Lambda$.

Since DSM obviously implies DSU, it certainly implies the uniqueness of Gibbs states. In fact, as Dobrushin and Shlosman showed, it implies analytic dependence. To be precise, if $\Phi$ and $\Psi$ are a pair of shift-invariant, finite range Gibbs potentials and if DSM holds for the local specification associated with $\Phi$, then there is a $\delta>0$ such that DSM holds for the local specification corresponding to each of Gibbs potentials $\Phi+t \Psi,|t|<\delta$, and the mapping $t \in(-\delta, \delta) \mapsto \mu_{\Phi+t \Psi} \in \mathfrak{M}_{1}(\Omega)$ is realanalytic in the sense that

$$
t \in(-\delta, \delta) \mapsto \mu_{\Phi+t \Psi}(\varphi) \in \mathbb{R}
$$

is real-analytic for each $\varphi \in \mathfrak{A}_{0}(\Omega)$.

Stochastic Dynamics. Let $\Phi$ be a shift-invariant, finite range Gibbs potential and $\mathfrak{E}=\left\{E_{\Lambda}\right\}_{\Lambda \in \mathfrak{F}}$ the corresponding local specification. In the course of this article, we will be dealing with several stochastic dynamics which are all connected to $\Phi$ by the property that each is reversible with respect to every $\mu \in \mathfrak{5}(\Phi)$. In order to describe these dynamics, we begin by saying how their generators act on $\mathfrak{A}_{0}(\Omega)$. To this end, let $\mathfrak{F} \ni Y \ni \mathbf{0}$ be given set, and, for each $\mathbf{k} \in \Gamma, \Lambda \in \mathfrak{F}$, and $\omega \in \Omega$, define the operator $\mathscr{L}_{\mathbf{k}}^{Y, \Lambda, \omega}: \mathfrak{A}(\Omega) \rightarrow \mathfrak{U}(\Omega)$ by

$$
\mathscr{L}_{\mathbf{k}}^{Y, \Lambda, \omega} \varphi(\eta)=\left[E_{\Lambda \cap(\mathbf{k}+Y)} \varphi\right]\left(\eta_{\Lambda} \cdot \omega_{\Lambda \uparrow}\right)-\varphi_{\Lambda}(\eta \mid \omega) \quad \text { where } E_{\emptyset} \varphi \equiv \varphi .
$$

Next, for $\omega \in \Omega$ and $\Lambda \in \mathfrak{F}$, set

$$
\mathscr{L}^{Y, \Lambda, \omega} \varphi=\sum_{k \in \Gamma} \mathscr{L}_{\mathbf{k}}^{Y, \Lambda, \omega} \varphi, \quad \varphi \in \mathfrak{U}(\Omega) .
$$

Because $\mathscr{L}^{Y, \Lambda, \omega}$ is a bounded, Markov generator, the operators

$$
P_{t}^{Y, \Lambda, \omega} \equiv \exp \left[t \mathscr{L}^{Y, \Lambda, \omega}\right]
$$

are well-defined for each $t \in(0, \infty)$ and form a Markov semigroup on $\mathfrak{A}(\Omega)$. In fact, it is clear that $\mathfrak{A}_{\Lambda}(\Omega)$ is invariant under $\left\{P_{t}^{Y, \Lambda, \omega}: t \in(0, \infty)\right\}$. Furthermore, because

$$
\begin{aligned}
& -\int_{\Omega} \varphi(\xi)\left[\mathscr{L}^{Y, \Lambda, \omega} \psi\right](\xi) E_{\Lambda}^{\omega}(d \xi)=\mathscr{E}^{Y, \Lambda, \omega}(\varphi, \psi) \text { where } \\
& \mathscr{E}^{Y, \Lambda, \omega}(\varphi, \psi) \\
& \equiv \frac{1}{2} \sum_{\mathbf{k} \in \Lambda} \int_{\Omega}\left(\int_{\Omega}(\varphi(\eta)-\varphi(\xi))(\psi(\eta)-\psi(\xi)) E_{\Lambda \cap(\mathbf{k}+X)}^{\xi}(d \eta)\right) E_{\Lambda}^{\omega}(d \xi),
\end{aligned}
$$


it is an easy matter to see that

$$
\int_{\Omega} \varphi(\xi)\left[P_{t}^{Y, \Lambda, \omega} \psi\right](\xi) E_{\Lambda}^{\omega}(d \xi)=\int_{\Omega} \psi(\xi)\left[P_{t}^{Y, \Lambda, \omega} \varphi\right](\xi) E_{\Lambda}^{\omega}(d \xi)
$$

for all $t \in(0, \infty)$ and $\varphi, \psi \in \mathfrak{U}(\Omega)$. In particular, by taking $\psi \equiv 1$ in (1.3), one sees that $E_{\Lambda}^{\omega}$ is $\left\{P_{t}^{Y, \Lambda, \omega}: t \in(0, \infty)\right\}$-invariant, and elementary considerations enable one to check that it is the only such measure.

So far we have described dynamics involving only finitely many sites, whereas our main interest is in dynamics which involve the spins at all sites of $\Gamma$. Thus, once again, let $\mathfrak{F} \ni Y_{\ni} \mathbf{0}$ be given, and define $\mathscr{L}^{Y}: \mathfrak{H}_{0}(\Omega) \rightarrow \mathfrak{A}_{0}(\Omega)$ by

$$
\mathscr{L}^{\mathbf{Y}} \varphi=\sum_{\mathbf{k} \in \Gamma} \mathscr{L}_{\mathbf{k}}^{\mathbf{Y}} \varphi \text { where }\left[\mathscr{L}_{\mathbf{k}}^{\mathbf{Y}} \varphi\right](\eta) \equiv\left[E_{\mathbf{k}+Y} \varphi\right](\eta)-\varphi(\eta) .
$$

Although $\mathscr{L}^{Y}$ is no longer bounded, it is nonetheless well-known (e.g., see Theorem 3.9 in [L]) that our assumptions about $\Phi$ are more than enough to guarantee that there exists precisely one Markov semigroup $\left\{P_{t}^{Y}: t \in(0, \infty)\right\}$ on $\mathfrak{A}(\Omega)$ with the property that

$$
P_{t}^{Y} \varphi-\varphi=\int_{0}^{t} P_{s}^{Y} \circ \mathscr{L}^{Y} \varphi d s, \quad \varphi \in \mathfrak{A}_{0}(\Omega) \text { and } t \in(0, \infty) .
$$

In fact, $\left\{P_{t}^{Y}: t \in(0, \infty)\right\}$ preserves both $C(\Omega)$ and $C^{1}(\Omega)$; and so, after extending $\mathscr{L}^{Y}$ to $C^{1}(\Omega)$ by continuity, one can show from (1.4) that

$$
P_{t}^{Y} \varphi-\varphi=\int_{0}^{t} \mathscr{L}^{Y} \circ P_{s}^{Y} \varphi d s, \quad \varphi \in C^{1}(\Omega) \text { and } t \in(0, \infty) .
$$

Furthermore, because of uniqueness, one can easily see that for any exhaustion of $\Gamma$ by $\left\{\Lambda_{n}\right\}_{1}^{\infty}$ and $\left\{\omega_{n}\right\}_{1}^{\infty} \subset \Omega$,

$$
\left[P_{t}^{Y, \Lambda_{n}, \omega_{n}} \varphi\right](\eta) \rightarrow\left[P_{t}^{Y} \varphi\right](\eta) \quad \text { uniformly in }(t, \eta) \in(0, T] \times \Omega
$$

for each $T>0$ and $\varphi \in C(\Omega)$. In particular, if $\mu \in\left(\mathfrak{5}(\Phi)\right.$, then after taking $\Lambda=\Lambda_{n}$ in (1.3), integrating with respect to $\mu$, and passing to the limit as $n \rightarrow \infty$, we find that

$$
\int_{\Omega} \varphi(\xi)\left[P_{t}^{Y} \psi\right](\xi) \mu(d \xi)=\int_{\Omega} \psi(\xi)\left[P_{t}^{Y} \varphi\right](\xi) \mu(d \xi), \quad t \in(0, \infty) \text { and } \mu \in \mathbb{G}(\Phi)
$$

first for continuous $\varphi$ and $\psi$ and then for all $\varphi, \psi \in \mathfrak{U}(\Omega)$. Conversely, if $\mu$ is an element of $\mathfrak{M}_{1}(\Omega)$ for which the detailed balance condition

DB

$$
\mu\left(\varphi \mathscr{L}^{Y} \psi\right)=\mu\left(\psi \mathscr{L}^{Y} \varphi\right), \quad \varphi, \psi \in \mathfrak{A}_{0}(\Omega),
$$

holds, then one finds first that

$$
\begin{aligned}
\mu\left(\varphi \mathscr{L}^{Y} \psi\right) & =-\mathscr{E}_{\mu}^{Y}(\varphi, \psi), \quad \text { where } \\
\mathscr{E}_{\mu}^{Y}(\varphi, \psi) & \equiv \frac{1}{2} \sum_{\mathbf{k} \in \Gamma} \int_{\Omega^{2}}(\varphi(\eta)-\varphi(\omega))(\psi(\eta)-\psi(\omega)) E_{\mathbf{k}+Y}^{\omega}(d \eta) \mu(d \omega),
\end{aligned}
$$

for all $\varphi, \psi \in \mathfrak{U}_{0}(\Omega)$ and thence that $\mu \in(\mathfrak{5}(\Phi)$. In particular, since (1.5) certainly implies DB, we now known that, for any $\mu \in \mathfrak{M}_{1}(\Omega)$,

$$
(1.5) \Leftrightarrow \mu \in \mathbb{6}(\Phi) \Leftrightarrow \mathrm{DB} .
$$


Finally, we remark that if $\mu \in \mathfrak{5}(\Phi)$, then, because $P_{t}^{Y}$ is a Markov operator and (1.5) implies that $\mu$ is $P_{t}^{Y}$-invariant, $P_{t}^{Y}$ admits a unique extension as a contraction operator on $L^{p}(\mu)$ for each $p \in[1, \infty)$, and another application of (1.3) shows that the extension ${\overline{P_{t}^{Y}}}^{\mu}$ to $L^{2}(\mu)$ is a self-adjoint contraction. Thus, for each $\mu \in(\mathfrak{G}(\Phi)$, $\left\{{\overline{P_{t}^{Y}}}^{\mu}: t \in(0, \infty)\right\}$ is a strongly continuous semigroup of self-adjoint, Markov (i.e., non-negativity preserving) contractions on $L^{2}(\mu)$, and (cf. (1.6)) $\mathscr{E}_{\mu}^{Y}$ is the associated Dirichlet form.

Hypercontractivity. In general, the $L^{p}$-contraction property just mentioned is the best one can hope for when dealing with reversible Markov semigroups on a state space which is infinite dimensional. However, in special circumstances, it is possible for such a semigroup to be mildly smoothing. To be precise, we will say that the $v$-stationary, Markov semigroup $\left\{T_{t}: t \in(0, \infty)\right\}$ is hypercontractive with respect to the measure $v$ if there is a $c \in(0, \infty)$ with the property that

$\mathrm{H}$

$$
\left\|T_{t} f\right\|_{L^{q}(v)} \leqq\|f\|_{L^{p}(v)} \text { for all }(t, p, q) \in(0, \infty) \times(1, \infty)^{2}
$$

$$
\text { satisfying } p \leqq q \leqq 1+(p-1) e^{\frac{2 t}{c}} \text {. }
$$

When $v$ is a probability measure which is $\left\{T_{t}: t \in(0, \infty)\right\}$-reversing and $\mathscr{E}$ is the associated Dirichlet form, then L. Gross's integration lemma (cf. [G] or, for the general case, Corollary 6.1.17 in [DS]) says that $\mathrm{H}$ is equivalent to the logarithmic Sobolev inequality

$$
v\left(f^{2} \log f\right) \leqq c \mathscr{E}(f, f)+\|f\|_{L^{2}(v)}^{2} \log \|f\|_{L^{2}(v)}
$$

for all positive functions $f$.

The smallest $c$ for which LS holds is called the logarithmic Sobolev constant for the semigroup $\left\{T_{t}: t \in(0, \infty)\right\}$ relative to $v$. An interesting and important aspect of a logarithmic Sobolev inequality LS is that, in addition to being a coercivity statement, it contains an ergodicity statement. In particular, as was noted by B. Simon (cf. Corollary 6.1.17 in [DS]), LS implies the spectral gap estimate

SG $\quad \frac{1}{c} v(f, f) \leqq \mathscr{E}(f, f)$ or, equivalently, $\left\|T_{t} f-v f\right\|_{L^{2}(v)} \leqq e^{-\frac{t}{c}} v(f, f)^{\frac{1}{2}}$.

Conversely (cf. Theorem 6.1.22 in [DS]),

$$
\begin{gathered}
v\left(f^{2} \log |f|\right) \leqq \alpha \mathscr{E}(f, f)+\beta\|f\|_{L^{2}(v)}^{2}+\|f\|_{L^{2}(v)}^{2} \log \|f\|_{L^{2}(v)} \text { plus } v(f, f) \leqq \gamma \mathscr{E}(f, f) \\
\Rightarrow v\left(f^{2} \log |f|\right) \leqq(\alpha+(\beta+2) \gamma) \mathscr{E}(f, f)+\|f\|_{L^{2}(v)}^{2} \log \|f\|_{L^{2}(v)}
\end{gathered}
$$

With the preceding preliminaries in place, we can at last summarize the main conclusions which we will draw in this article.

1.8 Theorem. Let $\mathbb{E}$ be the local specification corresponding to a shift-invariant, finite range Gibbs potential $\Phi$, and use $\left\{P_{t}: t \in(0, \infty)\right\}$ to denote the associated Markov semigroup determined by (1.4) when $Y=\{\mathbf{0}\}$, and, for $\mu \in \mathfrak{G}(\Phi)$, let $\mathscr{E}_{\mu}$ be the Dirichlet form given by (1.6) when $Y=\{\mathbf{0}\}$.

a) If $\mathrm{DSU}(\mathrm{Y})$ holds for some $Y \in \mathfrak{F}$ with $\mathbf{0} \in Y$, then (cf. (1.4))

$$
\left\|P_{t}^{Y} f\right\| \leqq\|f \mid\| e^{-(1-\gamma)|Y| t} \quad \text { for } t \in(0, \infty) \text { and } f \in C^{1}(\Omega) .
$$


In particular, $\mathfrak{G}(\Phi)$ contains precisely one element $\mu$ and

$$
m_{\mu} \equiv \inf \left\{\mathscr{E}_{\mu}(f, f): \mu(f, f)=1\right\}>0
$$

or, equivalently,

$$
m_{\mu}=-\lim _{t \rightarrow \infty} \sup \left\{\left\|P_{t} f-\mu f\right\|_{L^{2}(\mu)}:\|f\|_{L^{2}(\mu)}=1\right\}>0 .
$$

b) If $\mathrm{DSM}(\mathrm{Y})$ holds for some $Y \in \mathfrak{F}$ with $\mathbf{0} \in Y$, then (cf. (1.1))

$\left\|P_{t}^{Y, \Lambda, \omega} f\right\| \leqq\left|\|f \mid\| e^{-(1-\gamma)|Y| t}, \quad t \in(0, \infty)\right.$ for all $\Lambda \in \mathfrak{F}, f \in \mathfrak{A}_{\Lambda}(\Omega)$, and $\omega \in \Omega$.

In particular, $\mathrm{DSM}(\mathrm{Y})$ not only implies $\mathrm{DSU}(\mathrm{Y})$ and therefore that $\mathbf{5}(\Phi)$ contains only one element, but also it implies DSM for some $M \in(0, \infty)$. Conversely, DSM for some $M \in(0, \infty)$ implies DSM(Y) for all sufficiently large $Y \in \mathfrak{F}$.

c) For each $\omega \in \Omega$ and $\Lambda \in \mathfrak{F}$, let $c(\omega, \Lambda)$ be the standard logarithmic constant (cf. $\mathrm{SLS}) c\left(E_{\Lambda}^{\omega}\right)$ for $E_{\Lambda}^{\omega}$. Then the following conditions are equivalent to each other:

(i) $\mathfrak{E}$ satisfies DSM for some $M \in(0, \infty)$

(ii) $\inf \left\{\mathscr{E}^{\{0\}, \Lambda, \omega}(\varphi, \varphi): \Lambda \in \mathfrak{F}, \omega \in \Omega\right.$, and $\left.E_{\Lambda}^{\omega}(\varphi, \varphi)=1\right\}>0$

(iii) $\sup \{c(\omega, \Lambda): \Lambda \in \mathfrak{F}$ and $\omega \in \Omega\}<\infty$

(iv) There is a $c \in(0, \infty)$ such that $\left\|P_{t}^{\{0\}, \Lambda, \omega} f\right\|_{L^{q}\left(E_{A}^{\omega}\right)} \leqq\|f\|_{L^{p}\left(E_{A}^{\omega}\right)} \quad$ whenever $q=1+(p-1) e^{\frac{2 t}{c}}$ for every $\Lambda \in \mathfrak{F}, \omega \in \Omega, p \in(1, \infty), t \in(0, \infty)$ and $f \in \mathfrak{A}(\Omega)$

(v) There exist $\varepsilon>0$ and $K<\infty$ such that $\left\|P^{\{0\}, A, \omega} f-\mu f\right\|_{\mathrm{u}} \leqq K\|f\| \| e^{-\varepsilon t}, \quad t \in(0, \infty)$ for every $\Lambda \in \mathfrak{F}, \omega \in \Omega$, and $f \in C^{1}(\Omega)$.

Moreover, if any one of these holds and $\mu$ is the unique element of $(\mathfrak{b}(\Phi)$, then $c(\mu)<\infty$ and, for each $\theta \in(0,1)$, there is a $K_{\theta} \in(0, \infty)$ such that

$$
\left\|P_{t} \varphi-\mu \varphi\right\|_{\mathbf{u}} \leqq K_{\theta}\|\varphi\| e^{-\theta m_{\mu} t}, \quad t \in(0, \infty) \text { and } \varphi \in C^{1}(\Omega),
$$

where $m_{\mu}>0$ is the number defined in a) above.

1.15 Remark. As we will see in the following section, the contents of a) and b) are, more or less, a re-iteration of results obtained by Aizenman and Holley in [A\&H] combined with results in [SZ, 2]. As for the equivalences in (1.13), we have already shown in [SZ, 2] that the analogous assertions hold in the context of continuous spin systems (i.e., when $Q$ is replaced by a differentiable manifold and the dynamical system is an interacting diffusion). However, we found that the argument which we used in [SZ, 1] to check that i) implies iii) relies too heavily on Leibnitz's rule to be transferred to the context of discrete spin systems, and so we have been forced to adopt here an argument which derives from the one used in $[Z, 1]$ and $[Z, 2]$ and bears a close relation to ones employed recently by Maes and Shlosman in [MS]; and it turns out that this new argument is somewhat simpler than the one given in $[\mathrm{SZ}, 1]$. In particular, although we were unable to transfer the argument given in $[\mathrm{SZ}, 1]$ to the discrete spin context, it is an easy matter to transfer the reasoning used here to the continuous spin context in $[\mathrm{SZ}, 1]$. 
1.16 Remark. The equivalence ii) $\Leftrightarrow$ iii) in c) brings up an interesting and, so far as we know, unresolved question. Namely, although (as we pointed out in our discussion of hypercontractivity) LS always implies SG, it is not at all clear under what circumstances one can go the other direction. Indeed, the only examples (cf. [KS]) when we know for sure that the logarithmic Sobolev constant fails to be equal to half the reciprocal of the spectral gap are, in some sense, degenerate. Thus, there is a possibility that ii) $\Leftrightarrow$ iii) is a special case of a much more general phenomenon. In particular, is it possible that SG always implies LS in the context of compact, connected Riemannian manifolds?

1.17 Remark. In a good deal of the earlier literature on the Glauber dynamics associated with interacting systems of discrete spins, the authors have worked with a slightly different choice of the dynamics. In particular, these authors have often worked with the dynamics corresponding to an operator of the form

$$
\mathscr{L}=\sum_{\mathbf{k} \in \Gamma} c_{\mathbf{k}}(\omega) \nabla_{k}, \quad \text { where } c_{\mathbf{k}}(\omega)=\exp \left[\sum_{X \ni \mathbf{k}} U_{X}(\omega)\right] b_{\mathbf{k}}(\omega),
$$

where, for each $\mathbf{k} \in \Gamma$, the $b_{\mathbf{k}}$ is a positive function which does not depend on $\omega_{\mathbf{k}}$. However, because of the estimate in (2.4), it will be clear that all the estimates which we will derive here apply equally well to the semigroups corresponding to the operators in (1.18) so long as the $b_{\mathbf{k}}$ 's in (1.18) are uniformly positive.

\section{The Proof of Parts a) and b) of Theorem 1.8}

We begin with an argument which goes back to W. Sullivan [Sul] and has since then been adapted to various situations in [HS] and [A \& H]. However, the proof which we give below has some new features which we believe clarify what is happening.

2.1 Theorem. The condition $\operatorname{DSU}(\mathrm{Y})$ and $\operatorname{DSM}(\mathrm{Y})$ imply (1.9) and (1.12), respectively.

Proof. Let $Y \in \mathfrak{F}$ with $\mathbf{0} \in Y$ be given, and, depending on whether DSU(Y) or $\operatorname{DSM}(\mathrm{Y})$ holds, let $\Lambda=\Gamma$ or, respectively, $\omega \in \Omega$ and $\Lambda \in \mathfrak{F}$ be chosen and fixed. Next, for $\mathbf{j} \in \Gamma$, define

$$
\left[\mathbb{E}_{\mathbf{j}} \varphi\right](\eta)= \begin{cases}{\left[E_{\mathbf{j}+Y} \varphi\right](\eta)} & \text { if } \Lambda=\Gamma \\ {\left[E_{\Lambda \cap(\mathbf{j}+Y)} \varphi\right]\left(\eta_{\Lambda} \cdot \omega_{\Lambda \mathfrak{l}}\right)} & \text { if } \Lambda \in \mathfrak{F},\end{cases}
$$

and, recalling that $E_{\emptyset}$ is the identity, set

$$
\mathscr{L} \varphi=\sum_{\mathbf{j} \in \Gamma} \mathscr{L}_{\mathbf{j}} \varphi, \quad \text { where } \mathscr{L}_{\mathbf{j}} \varphi \equiv \mathbb{E}_{\mathbf{j}} \varphi-\varphi
$$

for $\varphi \in C^{1}(\Omega)$. It is then a relatively easy matter (e.g., see Theorem 3.9 in [L]) to check that there is a unique Markov semigroup $\left\{P_{t}: t>0\right\}$ on $C(\Omega)$ with the property that

$$
P_{t} \varphi-\varphi=\int_{0}^{t} P_{s} \circ \mathscr{L} \varphi d s, \quad t \in(0, \infty) \text { and } \varphi \in \mathfrak{A}_{0}(\Omega) .
$$


In fact, $C^{1}(\Omega)$ is $\left\{P_{t}: t>0\right\}$-invariant, and, when $\Lambda \in \mathfrak{F}, \mathfrak{U}_{\Lambda}(\Omega)$ is also $\left\{P_{t}: t>0\right\}-$ invariant. Thus, one has that

$$
\frac{d}{d t} P_{t} f=\mathscr{L} \circ P_{t} f \text { and }\left\|P_{t} f\right\| \leqq e^{\kappa t}\|f \mid\|, \quad t \in(0, \infty) \text { and } f \in C^{1}(\Omega),
$$

for some $\kappa \in(0, \infty)$.

Now let $\mathbf{k} \in \Lambda$ be given, note that

$$
\partial_{\mathbf{k}} \mathscr{L}_{\mathbf{j}} \varphi=-\partial_{\mathbf{k}} \varphi \quad \text { when } \mathbf{k} \in \mathbf{j}+Y
$$

and conclude that

$$
\frac{d}{d t}\left(\partial_{\mathbf{k}} P_{t} f\right)=\mathscr{L}^{(\mathbf{k})}\left(\partial_{\mathbf{k}} P_{t} f\right)-|Y| \partial_{\mathbf{k}} P_{t} f+R_{\mathbf{k}} P_{t} f,
$$

where

$$
\mathscr{L}^{(\mathbf{k})} \varphi \equiv \sum_{\mathbf{j} \notin-\mathbf{k}+Y} \mathscr{L}_{\mathbf{j}} \varphi \text { and } R_{\mathbf{k}} \varphi \equiv \sum_{\mathbf{j} \neq-\mathbf{k}+Y}\left(\partial_{k} \mathbb{E}_{\mathbf{j}}-\mathbb{E}_{\mathbf{j}} \partial_{\mathbf{k}}\right) \varphi
$$

In particular, $\mathscr{L}^{(\mathbf{k})}$ determines a unique Markov semigroup $\left\{P_{t}^{(\mathbf{k})}: t>0\right\}$ on $C(\Omega)$ such that

$$
P_{t}^{(\mathbf{k})} \varphi-\varphi=\int_{0}^{t} P_{s}^{(\mathbf{k})} \circ \mathscr{L}^{(\mathbf{k})} \varphi d s, \quad t \in(0, \infty) \text { and } \varphi \in \mathfrak{U}_{0}(\Omega)
$$

and so, for each $t \in(0, \infty)$,

$$
\frac{d}{d s}\left[e^{|Y| s} P_{t-s}^{(\mathbf{k})}\left(\partial_{\mathbf{k}} P_{s} f\right)\right]=e^{|Y| s} P_{t-s}^{(\mathbf{k})} \circ R_{\mathbf{k}} \circ P_{s} f, \quad s \in(0, t),
$$

from which it is an easy step to

$$
e^{|Y| t}\left\|\partial_{\mathbf{k}} P_{t} f\right\|_{\mathbf{u}} \leqq\left\|\partial_{\mathbf{k}} f\right\|_{\mathbf{u}}+\int_{0}^{t} e^{|Y| s}\left\|R_{\mathbf{k}} \circ P_{s} f\right\|_{\mathrm{u}} d s
$$

Finally, assuming that $f \in \mathfrak{U}_{0}(\Omega) \cap \mathfrak{U}_{\Lambda}(\Omega)$, summing the preceding over $\mathbf{k} \in \Lambda$, and, depending on which hypothesis has been made, applying DSU(Y) or DSM(Y), we arrive at

$$
e^{|Y| t}|| P_{t} f\||\leqq|\| f\left\|| + \gamma | Y \left|\int_{0}^{t} e^{|Y| s}\left\|P_{s} f \mid\right\| d s,\right.\right.
$$

from which the required estimate is an immediate consequence.

2.2 Corollary. DSU (Y) (and therefore also DSM(Y)) implies that $\mathfrak{0}(\Phi)$ contains precisely one element $\mu$. In addition, DSM(Y) implies DSM.

Proof. From (1.9), it is an easy matter to see that, for any $f \in C^{1}(\Omega)$,

$$
\lim _{t \rightarrow \infty} \sup _{\xi, \eta \in \Omega}\left|P_{t}^{Y} f(\xi)-P_{t}^{Y} f(\eta)\right|=0 .
$$

Hence, if $\alpha, \beta \in \mathfrak{M}_{1}(\Omega)$ are a pair of $\left\{P_{t}^{Y}: t>0\right\}$-invariant measures, then

$$
\alpha f-\beta f=\iint\left(P_{t}^{Y} f(\xi)-P_{t}^{Y} f(\eta)\right) \alpha(d \xi) \beta(d \eta) \rightarrow 0 \quad \text { as } t \rightarrow \infty ;
$$

and because every element of $\mathfrak{5}(\Phi)$ is $\left\{P_{t}^{Y}: t>0\right\}$-invariant, this proves that there is only one such element. 
In order to prove that DSM(Y) implies DSM, we apply Lemma 1.8 of [SZ, 2] to the semigroups $\left\{P_{t}^{Y, \Lambda, \omega}: t>0\right\}$ for two different $\omega$ 's and thereby conclude that DSM follows immediately from (1.12).

In oder to complete the proof of part a) in Theorem 1.8, we will use a simple comparison lemma which will also serve us well in the sequel.

2.3 Lemma. There is a $\kappa \in(0, \infty)$ such that, for any $Y \in \mathfrak{F}, \omega \in \Omega$, and $\Lambda \in \mathfrak{F}$, (cf. (1.2))

$$
e^{-\kappa|Y|} \mathscr{E}^{Y, \Lambda, \omega}(f, f) \leqq E_{\Lambda}^{\omega}\left|\nabla_{\Lambda} f\right|^{2} \leqq|Q|^{|Y|-1} e^{\kappa|Y|} \mathscr{E}^{Y, \Lambda, \omega}(f, f) .
$$

In particular, for each $\mu \in \mathfrak{b}(\Phi)$, (cf. (1.6) and the statement of Theorem 1.8)

$$
e^{-\kappa|Y|} \mathscr{E}_{\mu}^{Y}(f, f) \leqq \mathscr{E}_{\mu}(f, f) \leqq|Q|^{|Y|-1} e^{\kappa|Y|} \mathscr{E}_{\mu}^{Y}(f, f) .
$$

Proof. Because of the detailed balance condition DB, it is clear that (2.5) follows from (2.4). Similarly, if we show that there is a $\kappa \in(0, \infty)$ such that, for each $X \in \mathfrak{F}$ and $\mathbf{k} \in X$,

$$
\begin{aligned}
E_{X}\left(f-E_{X} f\right)^{2} & \leqq e^{\kappa|X|} \sum_{\mathbf{j} \in X} E_{X}\left(\partial_{\mathrm{j}} f\right)^{2} \text { and } \\
E_{X}\left(\partial_{\mathbf{k}} f\right)^{2} & \leqq|Q|^{|X|-1} e^{\kappa|X|} E_{X}\left(f-E_{X} f\right)^{2},
\end{aligned}
$$

then (2.4) will follow with this choice of $\kappa$ plus 1 . But, by elementary comparison of the measures $E_{X}^{\omega}\left\lceil Q^{X}\right.$ with $v_{0}^{X}$, we see that (2.6) reduces to proving that

$$
v_{0}^{X}\left(f-v_{0}^{X} f\right)^{2} \leqq|X| \sum_{\mathbf{j} \in X} v_{0}^{X}\left(\partial_{\mathbf{j}} f\right)^{2} \text { and } v_{0}^{X}\left(\partial_{\mathbf{k}} f\right)^{2} \leqq|Q|^{|X|-1} v_{0}^{X}\left(f-v_{0}^{X} f\right)^{2}
$$

for all $f \in \mathfrak{H}_{X}(\Omega)$. Finally, to prove the first assertion in (2.7), let $\left\{\mathbf{j}_{1}, \ldots, \mathbf{j}_{|X|}\right\}$ be an enumeration of the elements of $X$, set $X_{0}=\emptyset, X_{m}=\left\{\mathbf{j}_{1}, \ldots, \mathbf{j}_{m}\right\}$ for $1 \leqq m \leqq|X|$, and $X_{m}^{\prime}=X \backslash X_{m}$ for $0 \leqq m \leqq|X|$, and note that

$$
\begin{aligned}
v_{0}^{X}\left(f-v_{0}^{X} f\right)^{2} & =\frac{1}{2} \iint(f(\xi)-f(\eta))^{2} v_{0}^{X}(d \xi) v_{0}^{X}(d \eta) \\
& \leqq \frac{|X|}{2} \sum_{m=1}^{|X|} \iint\left(\partial_{\mathrm{j}_{m}} f\left(\xi_{X_{m}} \cdot \eta_{X_{m^{\prime}}}\right)\right)^{2} v_{0}^{X}(d \xi) v_{0}^{X}(d \eta) \\
& =|X| \sum_{\mathrm{j} \in X} v_{0}^{X}\left(\partial_{\mathrm{j}} f\right)^{2} .
\end{aligned}
$$

On the other hand, to prove the second part of (2.7), note that

$$
\begin{aligned}
v_{0}^{X}\left(\partial_{\mathbf{k}} f\right)^{2} & =\frac{1}{2} \iint\left(f\left(\xi_{\mathbf{k}} \cdot \eta_{X \backslash\{\mathbf{k}\}}\right)-f(\eta)\right)^{2} v_{0}\left(d \xi_{\mathbf{k}}\right) v_{0}^{X}(d \eta) \\
& =\frac{1}{2} \int\left(\int_{\left\{\xi: \xi_{X \backslash\{\mathbf{k}\}}=\eta_{X \backslash \mathbf{k}\}}\right\}}(f(\xi)-f(\eta))^{2} v_{0}^{X}(d \xi)\right) v_{0}^{X}(d \eta) \\
& \leqq|Q|^{|X|-1} v_{0}^{X}\left(f-v_{0}^{X} f\right)^{2} .
\end{aligned}
$$

2.8 Corollary. DSU (Y) implies (1.10) (and therefore (1.11)). Moreover, DSM(Y) implies that

$$
\inf \left\{\mathscr{E}^{\{0\}}, \Lambda, \omega(\varphi, \varphi): \Lambda \in \mathfrak{F}, \omega \in \Omega \text {, and } E_{\Lambda}^{\omega}(\varphi, \varphi)\right\}>0 .
$$

Proof. From (1.9) and elementary spectral theory, we know that

$$
\inf \left\{\mathscr{E}_{\mu}^{Y}(\varphi, \varphi): \mu(\varphi, \varphi)=1\right\} \geqq(1-\gamma)|Y|
$$


Thus, by the left-hand inequality in (2.5), we see that $m_{\mu} \geqq(1-\gamma)|Y| e^{-\kappa|Y|}$. Similarly, (1.12) and the left-hand inequality in (2.4) lead to (2.9).

\section{Proof of Part c) of Theorem 1.8}

We have already seen in Corollary 2.8 that i) $\Rightarrow$ ii) in part c) of Theorem 1.8 . In addition, the implication iii) $\Rightarrow$ iv) is nothing by Gross's integration lemma, and the implications iv) $\Rightarrow v$ ) as well as v) $\Rightarrow$ i) and $v) \Rightarrow(1.14)$ are proved in exactly the same way as the corresponding implications in [SZ, 2] (cf. Lemma 2.9 and Lemma 1.8 in that article). Hence, all that remains is to prove that ii) $\Rightarrow$ i) and that i) $\&$ ii) $\Rightarrow$ iii); and the first of these is contained in the following.

\subsection{Lemma. Set}

$$
\|\Phi\|=\sum_{X \ni 0}\left\|\Phi_{X}\right\|_{\mathbf{u}}
$$

and let $\varepsilon>0$ be given. Then there exists an $M=M(\varepsilon, R,\|\Phi\|) \in(0, \infty)$ such that for any $\Lambda \in \mathfrak{F}$ and $\omega \in \Omega$ satisfying

$$
\mathscr{E}^{\{0\}, \Lambda, \omega}(\varphi, \varphi) \geqq \varepsilon \quad \text { whenever } E_{\Lambda}^{\omega}(\varphi, \varphi)=1,
$$

any non-empty subsets $X$ and $Y$ of $\Lambda$, and any $f \in \mathfrak{A}_{X}(\Omega)$ and $g \in \mathfrak{U}_{Y}(\Omega)$, one has

$$
E_{\Lambda}^{\omega}(f, g) \leqq\|f\|\|\| g \| e^{-\operatorname{Md}(X, Y)} .
$$

In particular, there is a $C=C(R, M) \in(0, \infty)$ with the property that, for each $\mathbf{k} \notin \Lambda$, $\emptyset \neq X \subseteq \Lambda$, and $f \in \mathfrak{A}_{X}(\Omega)$ (cf. DSU)

$$
\sup \left\{\left|E_{\Lambda}^{\eta} f-E_{\Lambda}^{\omega} f\right|:(\eta, \omega) \in \Omega^{2}(\mathbf{k})\right\} \leqq C e^{-M d(\mathbf{k}, X)}\||f|\| .
$$

Proof. Let $n$ be the largest element of $\mathbb{N}$ with the property that $d(X, Y) \geqq 2 n R$, set $\Lambda_{1}=\{\mathbf{j} \in \Lambda: d(\mathbf{j}, X) \leqq n R\}$, and take $\Lambda_{2}=\Lambda \backslash \Lambda_{1}$. Next, take $P_{t}=P_{t}^{\{0\}, \Lambda, \omega}$, $P_{t}^{1}=P_{t}^{\{0\}, \Lambda_{1}, \omega}$, and $P_{t}^{2}=P_{t}^{\{0\}, \Lambda_{2}, \omega}$. By Lemma 1.8 in [SZ, 1], we know that, for any $f \in \mathfrak{U}_{X}(\Omega)$ and $g \in \mathfrak{U}_{Y}(\Omega)$,

$$
\begin{aligned}
\left\|P_{t}(f g)-P_{t}^{1} f P_{t}^{2} g\right\|_{\mathrm{u}} & \leqq e_{n}(C t)\|f g\|, \\
\left\|P_{t}(f)-P_{t}^{1} f\right\|_{\mathrm{u}} & \leqq e_{n}(C t)\|f\|, \\
\text { and }\left\|P_{t}(g)-P_{t}^{2} g\right\|_{\mathrm{u}} & \leqq e_{n}(C t)\|g\|,
\end{aligned}
$$

where $C \in(0, \infty)$ depends only on $R$ and $\|\Phi\|$ and

$$
e_{n}(s) \equiv e^{s}-\sum_{m=0}^{n-1} \frac{s^{m}}{m !} \leqq e^{s}\left(\frac{s e}{n}\right)^{n} .
$$

Starting from (3.5) and the fact that $E_{\Lambda}^{\omega}$ is $\left\{P_{t}: t \in(0, \infty)\right\}$-invariant, we obtain:

$$
\begin{aligned}
E_{\Lambda}^{\omega}(f g)= & E_{\Lambda}^{\omega}\left(P_{t}(f g)\right) \leqq E_{\Lambda}^{\omega}\left(P_{t}^{1} f P_{t}^{2} g\right)+e_{n}(C t)\|f g\| \\
\leqq & E_{\Lambda}^{\omega}\left(P_{t} f P_{t} g\right)+e_{n}(C t)\left(\|f\|_{\mathbf{u}}\|g\|+\|f\|\|g\|_{\mathbf{u}}+\|f g\|\right) \\
\leqq & E_{\Lambda}^{\omega} f E_{\Lambda}^{\omega} g+\left\|P_{t} f-E_{\Lambda}^{\omega} f\right\|_{L^{2}\left(E_{\Lambda}^{\omega}\right)}\|g\|_{L^{2}\left(E_{A}^{\omega}\right)} \\
& +e_{n}(C t)\left(\|f\|_{\mathbf{u}}\|g\|+\|f\|\|g\|_{\mathbf{u}}+\|f g\|\right)
\end{aligned}
$$


Hence, because

$$
\left\|P_{t} f-E_{\Lambda}^{\omega} f\right\|_{L^{2}\left(E_{\Lambda}^{\omega}\right)} \leqq e^{-\varepsilon t}\left\|f-E_{\Lambda}^{\omega} f\right\|_{L^{2}\left(E_{A}^{\omega}\right)}
$$

and, without loss of generality, we may assume that $f(\omega)=g(\omega)=0$ and therefore that $\|f\|_{\mathbf{u}} \leqq\|f\|$ and $\|g\|_{\mathbf{u}} \leqq\|g\|$, we conclude that

$$
E_{\Lambda}^{\omega}(f, g) \leqq\left(e^{-\varepsilon t}+3 e_{n}(C t)\right)|\|f|\||\|g\||,
$$

which, by taking $t$ proportional to $n$, leads to

$$
E_{\Lambda}^{\omega}(f, g) \leqq e^{-\delta n}\|f\|\|\mid\| g \|
$$

for a choice of $\delta>\mathbf{0}$ which depends only on $C$. Thus, we can take $M=\delta / 2 R$.

Finally, let $\mathbf{k} \notin \Lambda$ and set $Y=\{\mathbf{j} \in \Lambda:|\mathbf{j}-\mathbf{k}| \leqq R\}$. If $Y=\emptyset$, then there is nothing more to do. On the other hand, if $Y \neq \emptyset$, then for any $(\omega, \eta) \in \Omega^{2}(\mathbf{k})$ there is a positive $g \in \mathfrak{A}_{Y}(\Omega)$, with $\|g\|$ bounded independent of $\Lambda, \mathbf{k}$, and $(\omega, \eta)$, such that $E^{\eta} f=E^{\omega}(f g)$ for all $f \in \mathfrak{A}(\Omega)$. Hence, (3.3) implies that

$$
\left|E_{\Lambda}^{\omega} f-E_{\Lambda}^{\eta} f\right|=\left|E^{\omega}(f, g)\right| \leqq\left|\left\|g \left|\left\|e ^ { - M d ( X , Y ) } \left|\|f \mid\| \quad \text { for all } f \in \mathfrak{U}_{X}(\Omega) ;\right.\right.\right.\right.\right.
$$

and clearly (3.4) follows from this.

In view of the remarks preceding Lemma 3.1, the proof of part c) of Theorem 1.8 will be complete once we show that i) and ii) together imply iii). Thus, from now on, we will be assuming both that DSM holds and that there is an $\varepsilon>0$ such that

$$
\mathscr{E}^{\{0\}, \Lambda, \omega}(\varphi, \varphi) \geqq \varepsilon E^{\omega}(\varphi, \varphi) \text { for all } \Lambda \in \mathfrak{F}, \omega \in \Omega \text {, and } \varphi \in \mathfrak{U}(\Omega) \text {. }
$$

We begin with the observation that, without any changes, the argument used in Lemma 3.5 of $[\mathrm{SZ}, 1]$ applies equally well here and proves that one has, for each $\Lambda \in \mathfrak{F}$ and $\emptyset \neq X \subseteq \Lambda$, a standard logarithmic Sobolev inequality with (cf. (3.2))

$$
c\left(E_{\Lambda}^{\omega} ; X\right) \leqq c_{0} e^{4|X|\|\Phi\|}, \omega \in \Omega,
$$

where $c_{0}$ is the standard logarithmic Sobolev constant for the measure $v_{0}$ on $Q$. That is, $c_{0}$ is the smallest $c$ with the property that

$$
v_{0}\left(\varphi^{2} \log |\varphi|\right) \leqq c v_{0}\left(|\partial \varphi|^{2}\right)+v_{0} \varphi^{2} \log \left(v_{0} \varphi^{2}\right)^{\frac{1}{2}}
$$

for all $\varphi: Q \rightarrow \mathbb{R}$. (That $c_{0}<\infty$ is, perhaps, most easily seen as an application of the criterion given in (1.7).)

3.8 Remark. We next have to describe a procedure which will allow us to make effective use of the mixing guaranteed by DSM; and, in order to avoid confusion arising from overly complicated notation, we will restrict our attention to the proof that DSM implies that the standard logarithmic Sobolev constant $c(\mu)$ for the unique $\mu \in \mathfrak{G}(\Phi)$ can be estimated in terms of the dimension $d$, the range $R$, the quantity $\|\Phi\|$ in (3.2), and the positive number $M$ in DSM. We will then leave it to the reader to check for himself that our argument applies equally well to the Gibbs potential on $Q^{A}$ given by

$$
\Phi(\omega, \Lambda) \equiv\left\{\Phi_{X \cap \Lambda}(\cdot \mid \omega): \emptyset \neq X \subseteq \Lambda\right\} .
$$

Indeed, the only concern that one might have comes from the loss of shiftinvariance. On the other hand, it is not hard to dispel any such concern by simply checking that the only use of shift-invariance which we have made has been to simplify the statement of our hypotheses. 
Unfortunately, we must begin with the introduction of some more notation. In the first place, let $\left\{\mathbf{e}_{r}\right\}_{0}^{2^{d}-1}$ be the enumeration of $\{0,1\}^{d}$ in which $r=\sum_{i=1}^{d} e_{r}^{i} 2^{d-i}$. Next, let $L$ be a fixed (to be specified later) element of $\mathbb{Z}^{+}$, and set

$$
\begin{aligned}
& \Gamma_{r}=(L+2 R) \mathbf{e}_{r}+(2(L+2 R)) \mathbb{Z}^{d}, \quad Y_{\mathbf{k}}=\mathbf{k}+[0,2(L+R)]^{d} \cap \Gamma, \text { and } \\
& \Lambda_{r}=\bigcup_{\mathbf{k} \in \Gamma_{r}} Y_{\mathbf{k}} .
\end{aligned}
$$

Although the sets $\Lambda_{r}$ are infinite, because the blocks out of which each $\Lambda_{r}$ is built are separated from one another by a distance greater than the range of interaction, it is an easy matter to check that the transition probability function

$$
\omega \in \Omega \mapsto E_{\Lambda_{r}}^{\omega} \equiv \prod_{\mathbf{k} \in \Gamma_{r}} E_{Y_{\mathbf{k}}}^{\omega} \in \mathfrak{M}_{1}(\Omega)
$$

satisfies

$$
E_{\Lambda_{r}}^{\omega}\left(E_{X} f\right)=E_{\Lambda_{r}}^{\omega} f, \quad \text { for all } \omega \in \Omega, f \in \mathfrak{U}(\Omega) \text {, and } \emptyset \neq X \subseteq \Lambda_{r},
$$

and is therefore a regular conditional probability distribution of any $\mu \in \mathbb{6}(\Phi)$ given $\mathscr{F}_{\Lambda_{r} \mathfrak{l}}$. In particular, this means that

$$
\mu\left(E_{\Lambda_{r}} f\right)=\mu f \text { for all } f \in \mathfrak{U}(\Omega) \text { and } \mu \in \mathfrak{G}(\Phi),
$$

where we have adopted the notation $E_{\Lambda_{r}}$ to denote the Markov operator determined by the transition probability in (3.9). Moreover, because it is a product measure, a fundamental property of logarithmic Sobolev inequalities (cf. [G]) together with the estimate coming from (3.7) Lemma says that

$$
c_{L} \equiv \sup \left\{c\left(E_{\Lambda_{r}}^{\omega} ; \Lambda_{r}\right): \omega \in \Omega \text { and } 0 \leqq r \leqq 2^{d}-1\right\}<\infty .
$$

In order to get beyond the conclusion reached in (3.11), we introduce the Markov operators $\Pi_{n}: \mathfrak{U}(\Omega) \rightarrow \mathfrak{U}(\Omega)$ defined inductively so that $\Pi_{0}$ is the identity map and, for $n \in \mathbb{Z}^{+}$,

$$
\Pi_{n+1}=E_{\Lambda_{n}} \circ \Pi_{n} \text { where } \Lambda_{n}=\Lambda_{r} \text { if } n \equiv r \bmod 2^{d} .
$$

As is easy to check, for each $\Lambda \in \mathfrak{F}$,

$$
\Pi_{n}: \mathfrak{A}_{\Lambda}(\Omega) \rightarrow \mathfrak{U}_{\Lambda \cup \hat{\partial}_{n} \Lambda} \text { where } K=2 L+3 R+1 .
$$

In addition, by repeated application of (3.10), we know that

$$
\mu\left(\Pi_{n} f\right)=\mu f \text { for all } n \in N, f \in \mathfrak{U}(\Omega) \text {, and } \mu \in \mathfrak{G}(\Phi) .
$$

Moreover, if $f$ is a positive element of $\mathfrak{A}_{0}(\Omega)$ and we set $f_{n}=\left(\Pi_{n} f^{2}\right)^{\frac{1}{2}}$, then, by (3.10) and (3.11), we have that

$$
\begin{aligned}
\mu\left(f_{n-1}^{2} \log f_{n-1}\right) & =\mu\left(E_{\Lambda_{n-1}}\left(f_{n-1}^{2} \log f_{n-1}\right)\right) \\
& \leqq \mu\left(c_{L} E_{\Lambda_{n-1}}\left|\nabla_{A_{n-1}} f_{n-1}\right|^{2}+E_{\Lambda_{n-1}} f_{n-1}^{2} \log \left(E_{\Lambda_{n-1}} f_{n-1}^{2}\right)^{\frac{1}{2}}\right) \\
& =c_{L} \mu\left(\left|\nabla_{A_{n-1}} f_{n-1}\right|^{2}\right)+\mu\left(f_{n}^{2} \log f_{n}\right) ;
\end{aligned}
$$

from which we obtain

$$
\left.\mu\left(f^{2} \log f\right) \leqq\left. c_{L} \sum_{m=0}^{n-1} \mu\left(\mid \nabla_{\Lambda_{m+1}}\left(\Pi_{m} f^{2}\right)^{\frac{1}{2}}\right)\right|^{2}\right)+\mu\left(\Pi_{n} f^{2} \log \left(\Pi_{n} f^{2}\right)^{\frac{1}{2}}\right)
$$

for all $n \in \mathbb{Z}^{+}$and positive $f \in \mathfrak{A}_{0}(\Omega)$. 
It is now possible to explain the strategy which underlies our proof. Namely, we will show that, as a consequence of the exponential mixing provided by DSM, the $L$ can be chosen so that there exists a $C \in(0, \infty)$ and $\lambda \in[0,1)$ with the property that, for all positive $f \in \mathfrak{U}_{0}(\Omega)$,

$$
\mu\left(\left|\nabla\left(\Pi_{n} f^{2}\right)^{\frac{1}{2}}\right|^{2}\right) \leqq C \lambda^{n} \mu\left(|\nabla f|^{2}\right), \quad n \in \mathbb{Z}^{+},
$$

where $\mu$ is the unique element of $\mathfrak{( 5}(\Phi)$. Notice that, in conjunction with (3.12) and the fact that $m_{\mu}>0,(3.13)$ is all that we need. Indeed, given a positive $f \in \mathfrak{U}_{0}(\Omega)$, it not only shows that

$$
\mu\left(\Pi_{n} f^{2}, \Pi_{n} f^{2}\right) \leqq \frac{1}{m_{\mu}} \mu\left(\left|\nabla\left(\Pi_{n} f^{2}\right)\right|^{2}\right) \rightarrow 0 \quad \text { as } n \rightarrow \infty,
$$

but also that

$$
\sum_{m=0}^{\infty} \mu\left(\left|\nabla_{A_{m+1}}\left(\Pi_{m} f^{2}\right)^{\frac{1}{2}}\right|^{2}\right) \leqq \frac{C}{1-\lambda} \mu\left(|\nabla f|^{2}\right)
$$

and therefore, after letting $n \rightarrow \infty$ in (3.12), one finds that $c(\mu) \leqq \frac{C c_{\mathrm{L}}}{1-\lambda}$.

The main step in the proof of (3.13) is taken with the aid of the following somewhat tedious computation.

3.14 Lemma. For $\Lambda \in \mathfrak{F}$, define $\rho_{\Lambda}: \Omega^{2} \mapsto(0, \infty)$ by (cf. (1.2))

$$
\rho_{\Lambda}(\eta \mid \omega)=\frac{\exp \left[-U_{\Lambda}\left(\eta_{\Lambda} \mid \omega\right)\right]}{Z_{\Lambda}(\omega)} .
$$

Next, for $\mathbf{j} \notin \Lambda$ and $y \in Q$, set

$$
R_{\Lambda, \mathbf{j}}(\omega \mid y)=\frac{\rho_{\Lambda}\left(\omega \mid \omega^{\mathbf{j}} \cdot y\right)}{\rho_{\Lambda}(\omega \mid \omega)}
$$

where $\omega^{\mathbf{j}} \cdot y$ is the element of $\Omega$ which coincides with $\omega$ on $\Gamma \backslash\{\mathbf{j}\}$ and has $\mathbf{j}^{\text {th }}$ coordinate equal to $y$. Finally, for $\emptyset \neq X \subseteq \Lambda$, define

$$
\begin{aligned}
& \mathfrak{R}(\Lambda, X, \mathbf{j}) \\
= & \sup \left\{\left|E_{X}^{\xi}\left(R_{\Lambda, \mathbf{j}}(\cdot \mid y)\right)-E_{X}^{\eta}\left(R_{\Lambda, \mathbf{j}}(\cdot \mid y)\right)\right|: y \in Q \text { and }(\xi, \eta) \in \Omega^{2} \text { with } \xi_{\Lambda \uparrow}=\eta_{\Lambda \uparrow}\right\} .
\end{aligned}
$$

Then, for any positive $f \in \mathfrak{U}_{0}(\Omega)$ with the property that, for each $\omega \in \Omega, f_{\Lambda}(\cdot \mid \omega) \in$ $\mathfrak{U}_{\Lambda \backslash X}(\Omega)$, one has that (cf. (3.6))

$$
\left|\nabla_{\mathbf{j}}\left(E_{\Lambda}^{\omega} f^{2}\right)^{\frac{1}{2}}\right| \leqq 2 e^{2\|\Phi\|}\left(\left[\left(E_{\emptyset}+E_{\mathbf{j}}\right) \circ E_{\Lambda}\left|\nabla_{\mathbf{j}} f\right|^{2}\right](\omega)\right)^{\frac{1}{2}}+\frac{2 \mathfrak{R}(\Lambda, X, \mathbf{j})}{\varepsilon} E_{\Lambda}^{\omega}(f, f)^{\frac{1}{2}}
$$

Proof. Note that

$$
\begin{aligned}
\left|\left[\nabla_{\mathbf{j}}\left(E_{\Lambda} f^{2}\right)^{\frac{1}{2}}\right](\omega)\right| & =\left|\int_{Q}\left[\left(E_{\Lambda} f^{2}(\omega)\right)^{\frac{1}{2}}-\left(E_{\Lambda} f^{2}\left(\omega^{\mathbf{j}} \cdot y\right)\right)^{\frac{1}{2}}\right] v_{0}(d y)\right| \\
& \leqq \int_{Q} \frac{\left|E_{\Lambda} f^{2}(\omega)-E_{\Lambda} f^{2}\left(\omega^{\bullet} \cdot y\right)\right|}{\left(E_{\Lambda} f^{2}(\omega)\right)^{\frac{1}{2}}+\left(E_{\Lambda} f^{2}\left(\omega^{\mathbf{j}} \cdot y\right)\right)^{\frac{1}{2}}} v_{0}(d y) \leqq I_{\Lambda}(f, \omega)+J_{\Lambda}(f, \omega),
\end{aligned}
$$


where

$$
I_{\Lambda}(f, \omega)=\int_{Q} \frac{\left|A_{\Lambda}(f, \omega, y)\right|}{D_{\Lambda}(f, \omega, y)} v_{0}(d y) \text { and } J_{\Lambda}(f, \omega)=\int_{Q} \frac{\left|B_{\Lambda}(f, \omega, y)\right|}{D_{\Lambda}(f, \omega, y)} v_{0}(d y)
$$

with

$$
\begin{aligned}
& A_{\Lambda}(f, \omega, y)=\int_{Q^{\Lambda}} \rho_{\Lambda}\left(x^{\Lambda} \mid \omega^{\mathbf{j}} \cdot y\right)\left[f_{\Lambda}^{2}\left(x^{\Lambda} \mid \omega\right)-f^{2}\left(x^{\Lambda} \mid \omega^{\mathbf{j}} \cdot y\right)\right] v_{0}^{\Lambda}\left(d x^{\Lambda}\right), \\
& B_{\Lambda}(f, \omega, y)=\int_{Q^{\Lambda}}\left[\rho_{\Lambda}\left(x^{\Lambda} \mid \omega\right)-\rho_{\Lambda}\left(x^{\Lambda} \mid \omega^{\mathbf{j}} \cdot y\right)\right] f_{\Lambda}^{2}\left(x^{\Lambda} \mid \omega\right) v_{0}^{\Lambda}\left(d x^{\Lambda}\right),
\end{aligned}
$$

and

$$
D_{A}(f, \omega, y)=\left(E_{\Lambda} f^{2}(\omega)\right)^{\frac{1}{2}}+\left(E_{A} f^{2}\left(\omega^{\mathbf{j}} \cdot y\right)\right)^{\frac{1}{2}} .
$$

By Schwarz's inequality, the triangle inequality, and the fact that $R_{\Lambda, \mathbf{j}} \leqq e^{2\|\Phi\|}$, we see that

$$
\begin{aligned}
\left|A_{\Lambda}(f, \omega, y)\right| \leqq & \left(\int_{Q^{\Lambda}} \rho_{\Lambda}\left(x^{\Lambda} \mid \omega^{\mathbf{j}} \cdot y\right)\left[f_{\Lambda}\left(x^{\Lambda} \mid \omega\right)+f\left(x^{\Lambda} \mid \omega^{\mathbf{j}} \cdot y\right)\right]^{2} v_{0}^{\Lambda}\left(d x^{\Lambda}\right)\right)^{\frac{1}{2}} \\
& \times\left(\int_{Q^{\Lambda}} \rho_{\Lambda}\left(x^{\Lambda} \mid \omega^{\mathbf{j}} y\right)\left[f_{\Lambda}\left(x^{\Lambda} \mid \omega\right)-f_{\Lambda}\left(x^{\Lambda} \mid \omega^{\mathbf{j}} \cdot y\right)\right]^{2} v_{0}^{\Lambda}\left(d x^{\Lambda}\right)\right)^{\frac{1}{2}} \\
\leqq & \left(e^{\|\Phi\|}\left(E_{\Lambda} f^{2}(\omega)\right)^{\frac{1}{2}}+\left(E_{\Lambda} f^{2}\left(\omega^{\mathbf{j}} y\right)\right)^{\frac{1}{2}}\right) \\
& \times\left(\int_{Q^{\Lambda}} \rho_{\Lambda}\left(x^{\Lambda} \mid \omega^{\bullet} \cdot y\right)\left[\left(\nabla_{\mathbf{j}} f\right)_{\Lambda}\left(x^{\Lambda} \mid \omega\right)-\left(\nabla_{\mathbf{j}} f\right)_{\Lambda}\left(x^{\Lambda} \mid \omega^{\mathbf{j}} \cdot y\right)\right]^{2} v_{0}^{\Lambda}\left(d x^{\Lambda}\right)\right)^{\frac{1}{2}} \\
\leqq & e^{\|\Phi\|} D_{\Lambda}(f, \omega, y)\left(\left[E_{\Lambda}\left|\nabla_{\mathbf{j}} f\right|^{2}\right]^{\frac{1}{2}}(\omega)+e^{\|\Phi\|}\left[E_{\Lambda}\left|\nabla_{\mathbf{j}} f\right|^{2}\right]^{\frac{1}{2}}\left(\omega^{\mathbf{j}} \cdot y\right)\right),
\end{aligned}
$$

and therefore, since $\rho_{\{\mathrm{j}\}}(\cdot \mid \omega) \geqq e^{-2\|\Phi\|}$, that

$$
I_{\Lambda}(f, \omega) \leqq e^{\|\Phi\|}\left[E_{\Lambda}\left|\nabla_{\mathbf{j}} f\right|^{2}\right](\omega)^{\frac{1}{2}}+e^{2\|\Phi\|}\left[E_{\mathbf{j}} \circ E_{\Lambda}\left|\nabla_{\mathbf{j}} f\right|^{2}\right](\omega)^{\frac{1}{2}} .
$$

Turning to the estimate of $J_{A}(f, \omega)$, note that

$$
\begin{aligned}
\left|B_{\Lambda}(f, \omega, y)\right| & =\left|\int_{Q^{\Lambda}}\left[\rho_{\Lambda}\left(x^{\Lambda} \mid \omega\right)-\rho_{\Lambda}\left(x^{\Lambda} \mid \omega^{\mathbf{j}} y\right)\right]\left[f_{\Lambda}^{2}\left(x^{\Lambda} \mid \omega\right)-\left(E_{\Lambda}^{\omega} f\right)^{2}\right] v_{0}\left(d x^{\Lambda}\right)\right| \\
& =\left|\int_{\Omega}\left[R_{\Lambda, \mathbf{j}}(\eta \mid y)-1\right]\left[f_{\Lambda}^{2}(\eta \mid \omega)-\left(E_{\Lambda}^{\omega} f\right)^{2}\right] E_{\Lambda}^{\omega}(d \eta)\right| \\
& \leqq \sup _{\eta}\left|E_{X}^{\eta}\left(R_{\Lambda, \mathrm{j}}(\cdot \mid y)-1\right)\right| E_{\Lambda}^{\omega}\left(\left|f^{2}-\left(E_{\Lambda}^{\omega} f\right)^{2}\right|\right),
\end{aligned}
$$

where, in the final line, we have used the fact that $f_{\Lambda}(\cdot \mid \omega) \in \mathfrak{U}_{\Lambda \backslash X}(\Omega)$. Next, again by Schwarz,

$$
E_{\Lambda}^{\omega}\left(\left|f^{2}-\left(E_{\Lambda}^{\omega} f\right)^{2}\right|\right) \leqq 2\left(E_{\Lambda}^{\omega} f^{2}\right)^{\frac{1}{2}} E_{\Lambda}^{\omega}(f, f)^{\frac{1}{2}} \leqq 2 D_{\Lambda}(f, \omega, y) E_{\Lambda}^{\omega}(f, f)^{\frac{1}{2}},
$$

whereas

$$
\begin{aligned}
\left|E_{X}^{\eta}\left(R_{\Lambda, \mathbf{j}}(\cdot, y)-1\right)\right| & =\left|E_{X}^{\eta}\left(R_{\Lambda, \mathbf{j}}(\cdot \mid y)\right)-E_{\Lambda}^{\eta}\left(R_{\Lambda, \mathbf{j}}(\cdot \mid y)\right)\right| \\
& =\left|\int_{\Omega}\left[E_{X}^{\eta}\left(R_{\Lambda, \mathbf{j}}(\cdot, y)\right)-E_{X}^{\xi}\left(R_{\Lambda, \mathbf{j}}(\cdot, y)\right)\right] E_{\Lambda}^{\eta}(d \xi)\right| \leqq \mathfrak{R}(\Lambda, X, \mathbf{j}) .
\end{aligned}
$$


Hence, we' have arrived at

$$
J_{\Lambda}(f, \omega) \leqq 2 \Re(\Lambda, X, \mathbf{j}) E_{\Lambda}^{\omega}(f, f)^{\frac{1}{2}},
$$

which, in conjunction with (3.6), completes the proof of (3.16).

The form in which we will apply Lemma 3.14 is given in the following.

3.17 Lemma. There is a $K=K(\varepsilon,\|\Phi\|, M, R) \in(0, \infty)$ (cf. (3.6) and DSM) such that, for all $n \in \mathbb{N}, \mathbf{j} \notin \Lambda_{n}$, and positive $f \in \mathfrak{H}_{0}(\Omega)$,

$\mu\left|\nabla_{\mathbf{j}}\left(\Pi_{n+1} f^{2}\right)^{\frac{1}{2}}\right|^{2} \leqq K\left(\sum_{|\mathbf{i}-\mathbf{j}|<\rho} \mu\left|\nabla_{\mathbf{i}}\left(\Pi_{n} f^{2}\right)^{\frac{1}{2}}\right|^{2}+e^{-2 M \rho} \sum_{|\mathbf{j}-\mathbf{i}| \leqq 2 L+3 R} \mu\left|\nabla_{\mathbf{i}}\left(\Pi_{n} f^{2}\right)^{\frac{1}{2}}\right|^{2}\right)$

for every $\rho \in \mathbb{N}$. In particular,

$$
\mu\left|\nabla_{\mathbf{j}}\left(\Pi_{n+1} f^{2}\right)^{\frac{1}{2}}\right|^{2} \leqq K \sum_{|\mathbf{i}-\mathbf{j}| \leqq 2 L+3 R} \mu\left|\nabla_{\mathbf{i}}\left(\Pi_{n} f^{2}\right)^{\frac{1}{2}}\right|^{2} .
$$

Proof. First observe that, by construction, there is a unique $\mathbf{l} \in \Gamma_{n}$ such that $\mathbf{j} \in \partial_{R} Y_{\mathrm{I}}$. Next, let $f$ be a positive element of $\mathfrak{A}_{0}(\Omega)$, choose $A \in \mathfrak{F}$ so that $\mathbf{I} \notin A \subseteq \Gamma_{n}$ and

$$
\Pi_{n} f^{2}=E_{A} \circ E_{Y} f^{2}, \quad \text { where } Y=Y_{1} \text { and } \Lambda=\bigcup_{\mathbf{k} \in A} Y_{\mathbf{k}},
$$

and set $g=\left(\Pi_{n} f^{2}\right)^{\frac{1}{2}}$. Because $\mathfrak{R}(\Lambda, \Lambda, \mathbf{j})=0,(3.15)$ applied to $g$ (with $X=\emptyset$ ) leads to

$$
\mu\left|\nabla_{\mathbf{j}}\left(\Pi_{n+1} f^{2}\right)^{\frac{1}{2}}\right|^{2} \leqq 8 e^{4\|\Phi\|} \mu\left|\nabla_{\mathbf{j}}\left(E_{Y} g^{2}\right)^{\frac{1}{2}}\right|^{2} .
$$

Next, set $X=\{\mathbf{i} \in Y:|\mathbf{i}-\mathbf{j}|<\rho\}$ and $h=\left(E_{X} g^{2}\right)^{\frac{1}{2}}$. Then, again by (3.15), but this time applied to $h$ (with $\Lambda=Y$ ), we see that

$$
\mu\left|\nabla_{\mathbf{j}}\left(E_{Y} g^{2}\right)^{\frac{1}{2}}\right|^{2} \leqq 16 e^{\|\Phi\|} \mu\left|\nabla_{\mathbf{j}} h\right|^{2}+8 \varepsilon^{-1} \mathfrak{R}(Y, X, \mathbf{j})^{2} \mu\left(E_{Y}(f, f)\right) .
$$

At the same time,

$$
\mu\left|\nabla_{\mathrm{j}} h\right|^{2} \leqq 8 e^{4\|\Phi\|} \mu\left|\nabla_{\mathrm{j}} g\right|^{2}+8 \varepsilon^{-1} e^{4\|\Phi\|} \mu\left|\nabla_{X} g\right|^{2},
$$

and

$$
\mu\left|\nabla_{Y} h\right|^{2} \leqq 16 e^{4\|\Phi\|} \sum_{\mathbf{i} \in Y \backslash X}\left(\mu\left|\nabla_{\mathbf{j}} g\right|^{2}+\mathbf{1}_{\partial_{R} X}(\mathbf{i}) \mu\left|\nabla_{X} g\right|^{2}\right) .
$$

Finally, since

$$
\left|\partial_{R} X\right| \leqq R(\rho+R)^{d-1} \quad \text { and } \quad \mathfrak{R}(Y, X, \mathbf{j}) \leqq e^{\|\Phi\|-M \rho},
$$

it is an easy matter to combine ii), (3.20), (3.21), and the preceding to arrive at (3.18). Moreover, (3.19) follows easily from (3.18) with $\rho=0$.

Proof of 3.13. As an application of (3.19) we see that, for any $n \in \mathbb{N}$,

$$
\mu\left|\nabla\left(\Pi_{n+1} f^{2}\right)^{\frac{1}{2}}\right|^{2} \leqq K(2 L+3 R)^{d} \mu\left|\nabla\left(\Pi_{n} f^{2}\right)^{\frac{1}{2}}\right|^{2} .
$$

Thus, in order to prove (3.13), it suffices for us to show that $L$ can be chosen so that

$$
\mu\left|\nabla\left(\Pi_{2^{a}} f^{2}\right)^{\frac{1}{2}}\right|^{2} \leqq \frac{1}{2} \mu|\nabla f|^{2} .
$$


To this end, set $\rho=\frac{L}{2^{d+1}}$, and let $\mathbf{j} \notin \Lambda_{2^{d}-1}$ be given. Then there is an $1 \leqq l \leqq 2^{d}-1$ such that $\left\{\mathbf{i}:|\mathbf{i}-\mathbf{j}| \leqq \frac{L}{2}\right\} \subseteq \Lambda_{l-1}$. After repeated application of (3.1), we obtain

$$
\begin{aligned}
\mu\left|\nabla_{\mathbf{j}}\left(\Pi_{2^{d}} f^{2}\right)^{\frac{1}{2}}\right|^{2} \leqq & K^{2^{d}-l} \sum_{|\mathbf{i}-\mathbf{j}| \leqq l \rho} \mu\left|\nabla_{\mathbf{i}}\left(\Pi_{l} f^{2}\right)^{\frac{1}{2}}\right|^{2} \\
& +e^{-2 M \rho} \sum_{n=1}^{2^{d}-l} K^{n} \sum_{|\mathbf{i}-\mathbf{j}| \leqq(2 L+3 R) n} \mu\left|\nabla_{\mathbf{i}}\left(\Pi_{2^{d}-n} f^{2}\right)^{\frac{1}{2}}\right|^{2} \\
\leqq & (4 K(2 L+3 R))^{d 2^{d}} \exp \left[-\frac{M L}{2^{d}}\right] \sum_{|\mathbf{i}-\mathbf{j}| \leqq(2 L+3 R) 2^{d}} \mu\left|\nabla_{\mathbf{i}} f\right|^{2},
\end{aligned}
$$

where, in the passage to the last line, we have first used the fact that $\nabla_{\mathbf{i}}\left(\Pi_{l} f^{2}\right)^{\frac{1}{2}}=0$ for all $|\mathbf{i}-\mathbf{j}| \leqq l \rho \leqq L$ and then applied (3.19). Hence, after summing over $\mathbf{j} \notin \Lambda_{2^{d}-1}$, we arrive at

$$
\mu\left|\nabla\left(\Pi_{2^{d}} f^{2}\right)^{\frac{1}{2}}\right|^{2} \leqq(4 K(2 L+3 R))^{(d+1) 2^{d}} \exp \left[-\frac{M L}{2^{d}}\right] \mu|\nabla f|^{2}
$$

from which (3.22) is an easy step.

As we said in the discussion containing (3.13), once we know that i) and ii) imply (3.13) for some $\lambda \in[0,1)$, the proof that i) \& ii) $\Rightarrow$ iii) is easy. Hence, we are done.

\section{References}

[A\&H] Aizenman, M., Holley, R.: Rapid convergence to equilibrium of stochastic Ising Models in the Dobrushin Shlosman regime. In Percolation Theory and Ergodic Theory of Infinite Particle Systems, Kesten, H. (ed.). IMS Volumes in Math. and Appl. vol. 8, pp. 1-11. Berlin, Heidelberg, New York: Springer 1987

[D\&S, 1] Deuschel, J.-D., Stroock, D.: Large Deviations. Pure Appl. Math. Series vol. 137, Boston: Academic Press, 1989

[Dob\&S, 1] Dobrushin, R.L., Shlosman, S.B.: Constructive criterion for the uniqueness of Gibbs field. In Statistical Physics and Dynamical Systems, Rigorous Results, (Fritz, J., Jaffe, A., Szasz, D.: (eds.), pp. 347-370. Boston: Birkhäuser 1985

[Dob\&S, 2] Dobrushin, R.L., Shlosman, S.B.: Completely analytical Gibbs fields. In Statistical Physics and Dynamical Systems, Rigorous Results, Fritz, J., Jaffe, A., Szasz, D. (eds.), pp. 371-403. Boston: Birkhäuser 1985

[Dob\&S, 3] Dobrushin, R.L., Shlosman, S.B.: Completely analytical interactions: Constructive description. J. Stat. Phys. 46, 983-1014 (1987)

[G] Gross, L.: Logarithmic Sobolev inequalities. Am. J. Math. 97, 1061-1083 (1976)

[HS] Holley, R., Stroock, D.: Applications of the stochastic Ising model to the Gibbs states. Commun. Math. Phys. 249-265 (1976)

[KS] Korzeniowski, A., Stroock, D.: An example in the theory of hypercontractive semigroups. Proc. A.M.S. 94, 87-90 (1985)

[L] Liggett, T.: Infinite Particle Systems. Grundlehren Series vol. 276, Berlin, Heidelberg, New York: Springer 1985

[MS] Maes, C., Shlosman, S.: Ergodicity of probabilistic automata. Commun. Math. Phys. 135, 233-551 (1991)

[Sul] Sullivan, W.: A unified existence and ergodic theorem for Markov e evolution of random fields. Z. Wahr. Verw. Geb. 31, 47-56 (1974) 
[SZ, 1] Stroock, D., Zegarlinski, B.: The logarithmic Sobolev inequality for continuous spin systems on a lattice. J. Funct. Anal. 104, 229-326 (1992)

[SZ, 2] Stroock, D., Zegarlinski, B.: The equivalence of the logarithmic Sobolev inequality and the Dobrushin-Shlosman mixing condition. Commun. Math. Phys. 144, 303-323 (1992)

$[\mathrm{Z}, 1]$ Zegarlinski, B.: Dobrushin uniqueness theorem and logarithmic Sobolev inequalities. J. Funct. Anal. 105, 77-111 (1992)

[Z, 2] Zegarlinski, B.: On log-Sobolev inequalities for infinite lattice systems. Lett. Math. Phys. 20, 173-182 (1990)

[Z, 3] Zegarlinski, B.: Log-Sobolev inequalities for infinite one-dimensional lattice systems. Commun. Math. Phys. 133, 147-162 (1990)

Communicated by Ya.G. Sinai 
